\title{
Web technologies in cartographic support of geological exploration
}

\author{
Loginov D.S. ${ }^{a *}$ \\ ${ }^{a}$ Target Horizon Inc., Moscow, Russian Federation; loginov@cartlab.ru \\ * Corresponding author
}

\begin{abstract}
:
Web technologies are now an integral part of the implementation of research work in various branches of science. The geological and geophysical direction is no exception, where the planning and execution of geological and geophysical surveys depend on the accuracy, reliability and relevance of the transmitted information.

The article deals with the possibilities of web technologies for cartographic support of geological exploration - a complex of scientific and production works, designed to determine the industrial significance of mineral deposits (ore, hydrocarbon, etc.). Examples are given of the use of geoportal solutions for the publication of data on the territory of study.

Taking into account the current level of web technologies development, a proprietary web-service was created to provide operational access to geodata during geological and geophysical work. The presented solution is implemented using PostgreSQL DBMS, PostGIS geospatial data processing module, Leaflet JavaScript library. The resulting interactive map allows to perform operational monitoring of field crews during seismic exploration, provides information on the stages of data processing and interpretation. Also it allows to implement a unified geoinformation space for joint work of specialists from different industries.
\end{abstract}

Keywords: Web maps, Cartographic support, Geological exploration, PostGIS, Leaflet

\section{Introduction}

Web technologies are nowadays an integral part of everyday life. Scientific research carried out in different branches of science and at their intersection is no exception. These areas include geological and geophysical work, which often goes hand in hand with cartography.

The emergence of GIS and its massive use for geological tasks allowed reaching a new qualitative level not only in geology and geophysics itself, but also in cartography in general. Rethinking the mapping processes, organisation of data access (including Big Data) is now reaching a new level in connection with the active implementation of web technologies into the scientific and practical work of geological and geophysical organisations.

The purpose of this article is to highlight the role of web technologies in the production process of geological and geophysical organisations whose tasks include the processing and interpretation of geophysical data during exploration work.

\section{About a unified geographic information space}

Understanding processes, predicting their development and occurrence in other areas becomes much easier when using a geospatial feature. Therefore, geodata is the link between the semantic meaning of geological models and their spatial reference.
In this regard, the creation of a unified geospatial space is closely related not only with the organization of spatial data infrastructure, but also with the production processes in the course of which that very information is created. Often in companies, professionals are faced with the lack of a common storage space for large amounts of geodata. As a result, information accumulates in disparate places, and it is not possible to add it into ongoing or future surveys of adjacent areas without losing time. In addition, it is not possible to fully utilise open data, including geological, geophysical and topographic study (knowledge about surveys location).

GIS is a powerful tool for solving the task. The author's research in the field of cartographic support of geological and geophysical works made has revealed the great role of GIS in the formation of a unified geographic information space (Loginov, 2019). In particular, it is proposed to use GIS projects not only as a means of working with data, but also as a means of storing and providing access to information to a wide range of users.

As geological and geophysical information is thematic data, the primary audience is geologists and geophysicists. Moreover, the larger the enterprise, the more users can work simultaneously in a single environment, imposing significant challenges in the technical organisation of data access. However, in the general terms, the concept of geological information 
support for geological exploration fulfils very specific objectives.

The software orientation is another difficulty that is seen in the use of GIS. Despite its widespread popularity, not all geologists and geophysicists are proficient in geoinformation technology. This somewhat narrows the range of users of GIS projects, which returns the issue of organising a unified geoinformation space to the technical aspect.

Considering that much of the information stored in geodatabases is valuable in terms of systematization and storage, it can be assumed that the main «core» of data has been identified. Such «core» is significantly smaller in terms of the information amount, because it characterizes the performed work at the same time in multiples and in full. In this way, it makes it possible to use other methods of providing access to data.

In this respect, web technologies come to the fore, as they are a tool that does not require geologists and geophysicist to have advanced knowledge of geoinformatics. The use of interactive maps requires no additional software installation (web browsers have definitely become universal, like MS Word) and their capabilities are intuitive. In addition, such maps can be used to visualize the very core of data that forms the framework of geological exploration work. Thus, the use of web technologies is a logical enhancement to providing unified access to data via GIS.

\section{Application of geoportals, GIS atlases for the analysis of area studies}

Geophysical study assessment is an important step in justifying the formulation of geological exploration.

Analysis of the geophysical exploration involves the use of a wide range of sources, including maps of geophysical study. These maps are showing the actual state of the exploration infrastructure: survey local and regional profiles, observation points, boreholes, boundaries of earlier survey, as well as subsoil use areas, etc. In addition, during the planning stage of field geophysical works, materials on the geological structure of the territory, topography of the area, etc. are thoroughly analyzed.

Currently, open data on geological and geophysical surveys are made available in the form of geoportals. These can be accessed either in the GIS or when creating your own interactive maps.

One of the key current sources of information is the One Geology geoportal. OneGeology is to make available Internet-accessible, scientifically-attributed data from countries participants.

The Geological Surveys of countries around the world are also preparing their own web-based solutions. Geological and geophysical data is provided through GIS atlases, which is a convenient solution for data access.

Since 2016, when more than a dozen GIS atlases of Geological Surveys of different European countries were analysed (Loginov, 2016), many different geoportals have appeared. Some of the previously analysed ones have been substantially updated. For example, Geological Survey of Italy switched to new visualization technology. Now it allows getting information both about geophysical phenomena and geological structure of the country (Figure 1). The data is accessible with the Geomapviewer and available as OGC service.

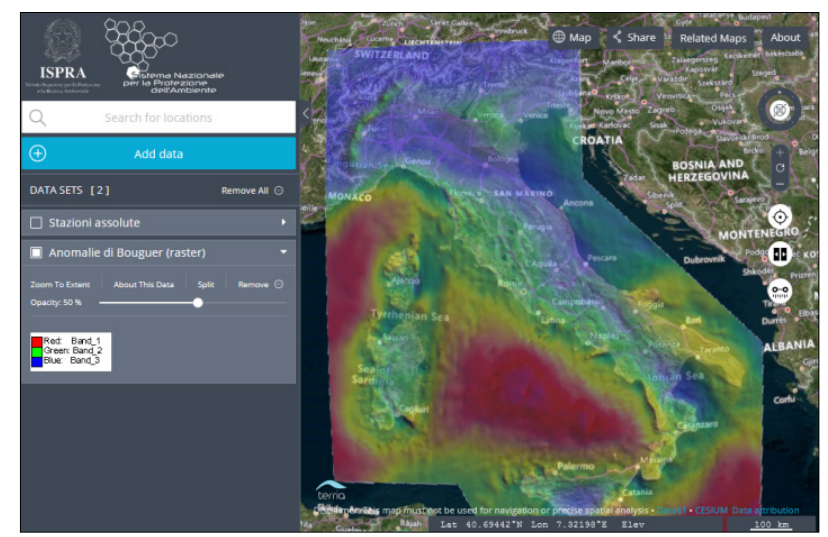

Figure 1. Work window of Geomapviewer with the Map of Bouger isoanomaly by ISPRA (C) Servizio geologico d'Italia, 2017

Map services based on open standarts has been prepared at the Mining and Geological Survey of Hungary (MBFSZ). The maps cover both geological and geophysical topics. The user-friendly interface of the individual maps provides information on seismic surveys and the distribution ofgravity and magnetic fields over the territory of Hungary. The only drawback is that all maps are available in separate windows, which makes it impossible to compare individual data layers (Figure 2).

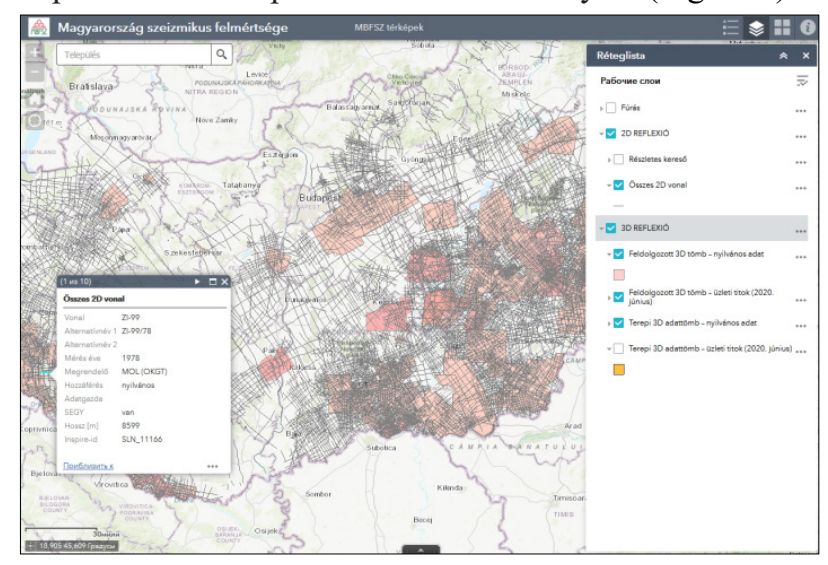

Figure 2. Work window of Mining and Geological Survey of Hungary with seismic study (C MBFSZ 2017

Geoportals are being actively developed in India. The Bhukosh from Geological Survey of India is a web application that acts as a client for spatial data infrastructure services. It is used to find, view, and query geospatial data and to integrate multiple sources into a single map view that can be easily navigated. The maps are predominantly geological in nature, but geophysics is represented by maps of the study (Figure 3).

The presented examples of web services are an integral part of the analysis of the study area's research coverage. Geoportals are simplifies the process of finding, connecting to, and displaying spatial data published 
through Web services. The use of OGS technology makes it possible to connect data via WMS, WFS to any GIS, which is also beneficial effect for the cartographic support of geological exploration works.

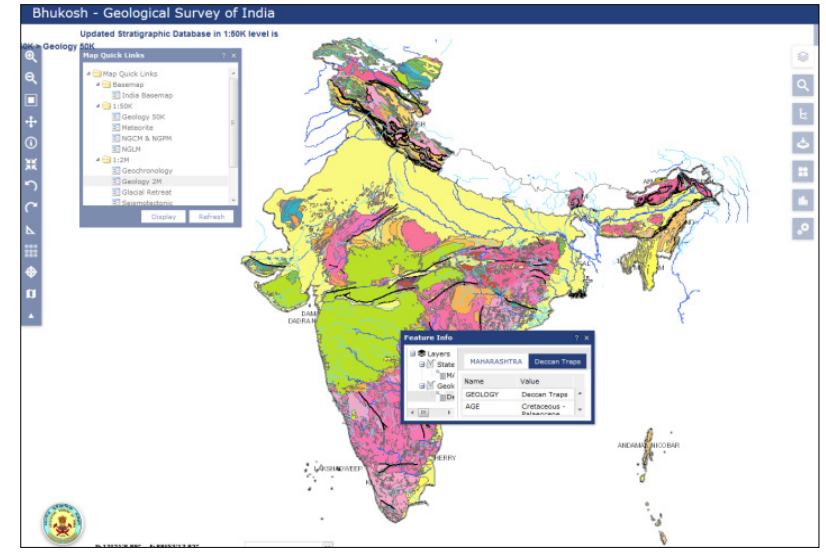

Figure 3. Work window of the Bhukosh by Geological Survey of India (C) GSI 2021

\section{Interactive maps for cartographic support of geological exploration}

Geological exploration is one of the most important areas of economic development in countries around the world. It is a complex of investigations and work designed to determine the commercial significance of mineral deposits (ore, hydrocarbon, etc.).

Three main stages of geological exploration are distinguished:

1) Regional geological studies of the subsoil and forecasting of mineral resources;

2) Prospecting and evaluation;

3) Subsoil exploration and development.

Each stage has its own work specifics, but what remains constant is that all surveys are conducted within a specific area of the earth's surface. At the regional exploration stage it is an oil and gas area or province, as well as a conventional contour; at the prospecting and evaluation stage it is a licensed subsoil area; at the exploration stage it is a deposit or a separate part of it.

A lot of geodata from different sources are used at each stage of the work. The GIS-atlases and geoportals presented earlier are some of them.

However, in addition to external data, internal data is also used. Internal data refers to a set of information created in the course of work by a limited number of persons, such as the team of a department or company. This is most common in prospecting and exploration work to clarify the geological structure and identify promising structures for further exploration.

The issue of developing your geoportal solutions nowadays requires qualified specialist in cartography, geoinformatics and programming. Taking into account the peculiarities of each discipline of thematic cartography, an additional requirement is an understanding of the processes and phenomena studied by a particular industry. For example, preparation of web maps to prospecting and appraisal work requires an understanding of the technological process of geological exploration.

Target Horizon Inc.'s Digital Cartography and GIS department is developing its own web applications to support geological and geophysical surveys, taking into account the current level of web technology development. Let us consider in more detail the result of testing the use of web-based technologies during regional and prospecting work to clarify the structure of sedimentary basins.

\subsection{Purpose of interactive map}

The interactive map to be created is intended for internal use within the company. It is a tool for visualization and exchange of intermediate information in geological and geophysical data processing and interpretation projects between specialists from different sectors.

One of the requirements: the use of free and open source software that allows the use of interactive mapping capabilities without additional software installation. Interactive maps are based on the use of open source software: PostgreSQL DBMS, PostGIS geospatial data processing module, Leaflet JavaScript library.

An important advantage of these technologies is that there is no need to install additional software, they are cross-platform, scalable and independent of closed products, which have high risks of stopping development support at an adequate level. Furthermore, the combination of PostgreSQL and Leaflet provides:

- $\quad$ spatial and attributive information retrieval and display;

- map scaling;

- performing cartometric works (distance measurements, navigation);

- $\quad$ import and export of *.kmz и *.kml files;

- move to a point at the specified coordinates;

- other operations.

The interactive maps can be used both in desktop mode using a browser and in mobile mode. Access to the electronic map is configurable for each user group, allowing the use of technologies to protect against unauthorised access.

A general flowchart for preparing interactive maps is shown in Figure 4. The presented scheme allows combining specialised thematic information with basic maps (topographic data, remote sensing data), as well as connecting survey information via WMS, WFS. These services are registered in the map code and represent a typical function. The organization of thematic data visualization is more interesting. It is described below. 


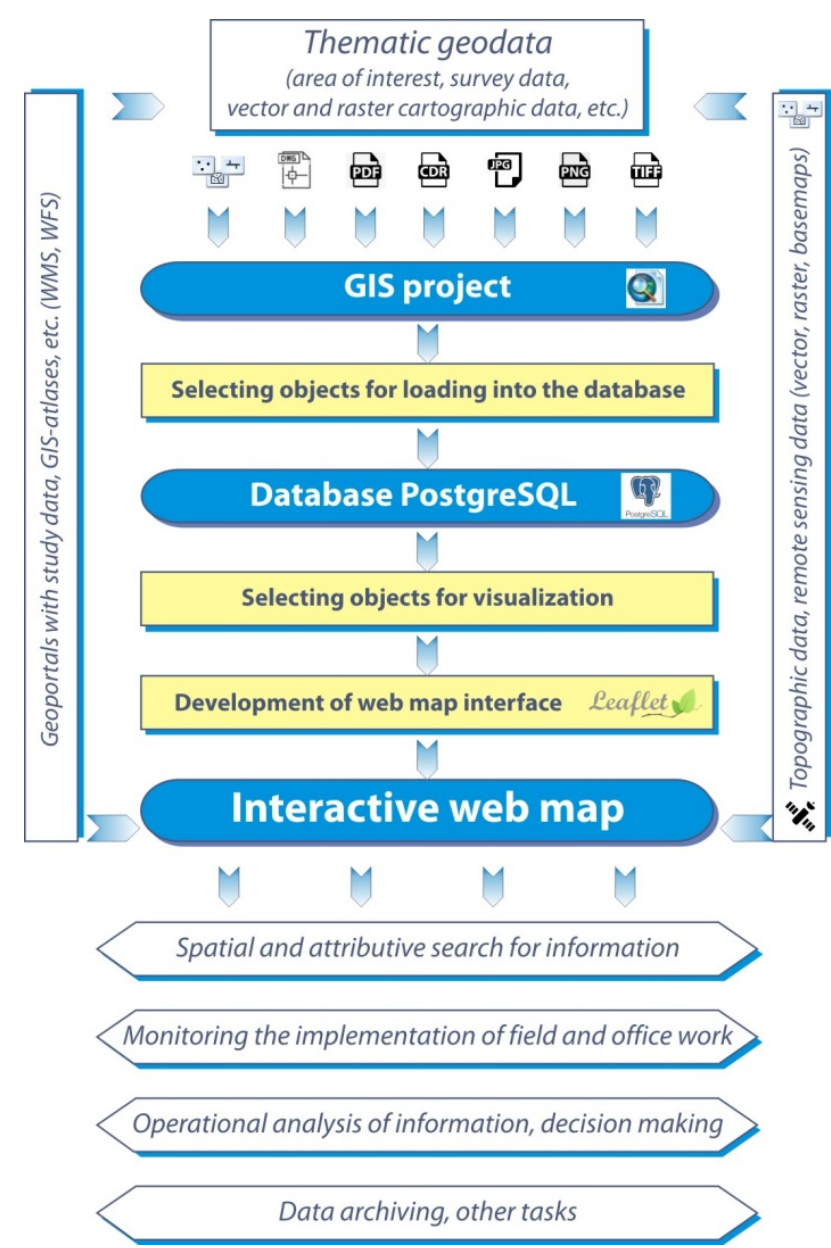

Figure 4. Technology of interactive web map preparing by Target Horizon Inc. (C) Loginov D.S., 2021

\subsection{Thematic content of interactive map}

Since the original information is presented in both raster and vector form, it is necessary to provide both data formats for the visualisation. In this regard, a combination of cashed and dynamic service capabilities is therefore applied.

The cached (static) data are georeferenced raster mosaics (tiles). This type is suitable for raster maps whose content will not change during operation, or will change only slightly. The requirement for static information is based on the fact that recalculation of tiles in the case of largescale data can take a long time. For infrequently updated data, therefore, the tiling is an effective tool. In addition, it allows the map content to be visualised much faster, as there is no waste of resources for data recalculating.

The cached type of operation is used in base maps (like Google maps), maps from WMS and WFS. The use of geological map with WMS from Bhukosh geoportal for own interactive map is shown in Figure 5.

Thematic maps can also become a background element of the interactive map. In particular, it is possible to connect pre-linked raster geological maps. Also, the tiled form is convenient for visualising specialised geological maps prepared in a GIS in accordance with the requirements of regulatory documents and the intended purpose of the work (Figure 6).

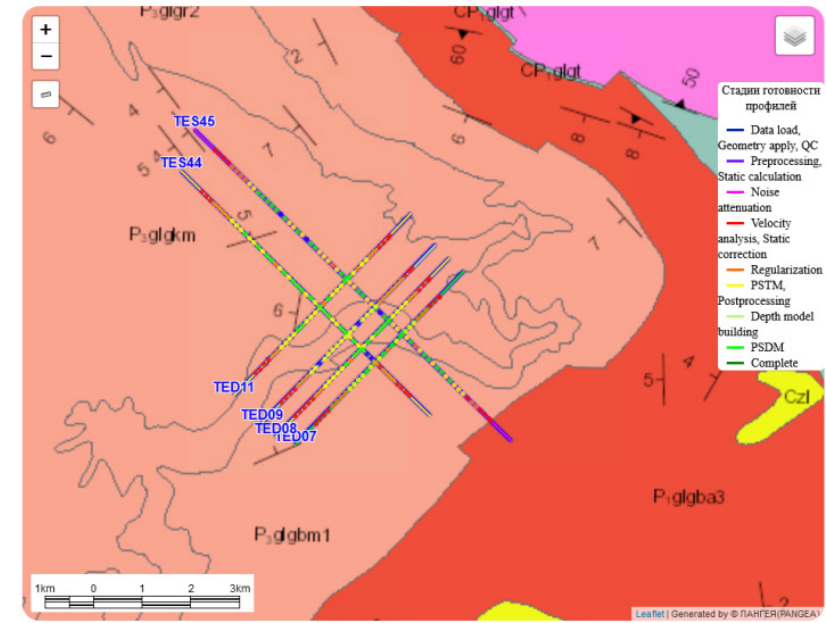

Figure 5. WMS and vector data using in interactive map. (C) Loginov D.S., 2021

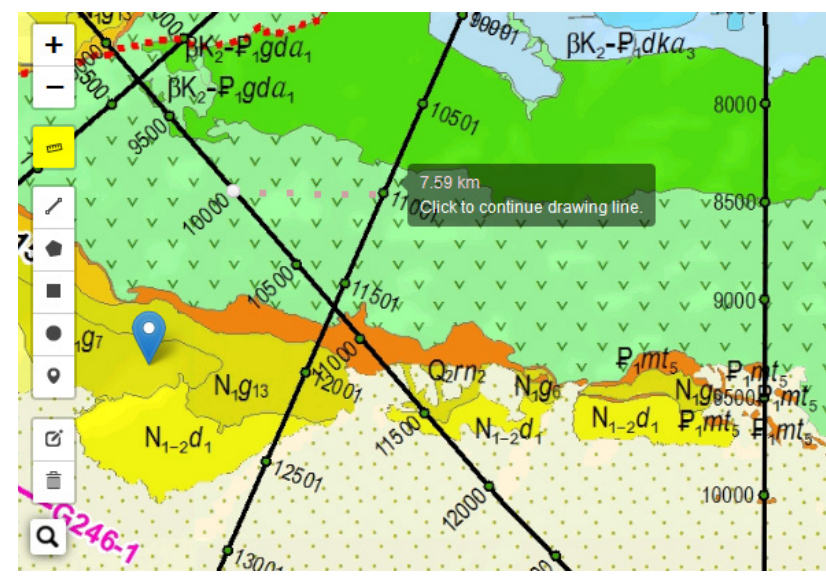

Figure 6. Work window of Target Horizon Inc. interactive map with specialized geological map. (C) Loginov D.S., 2021

Objects that are subject to more frequent updates are advantageously represented in a dynamic type. This includes survey data that come in as observations are made and data are processed. The data are provided from the GIS project, entered into the database, respecting the description of the coordinate system in which they are presented.

As a result, every time a map is scaled, the database is queried and features are redrawn. At the same time, layer visibility control, cartometric operations and attributive information, as well as changes in data and design, become available through interactive map manipualtion. In particular, Target Horizon Inc. specialists implemented the input and display of information on the processing of geological and geophysical data in a particular observation profile. The developed module is used to enter information about the current processing stage of 2D seismic profiles into the database. As a result, the colour of the line changes on the map, as well as statistical information in the graphs created on the basis of JavaScript libraries (Figure 7). 


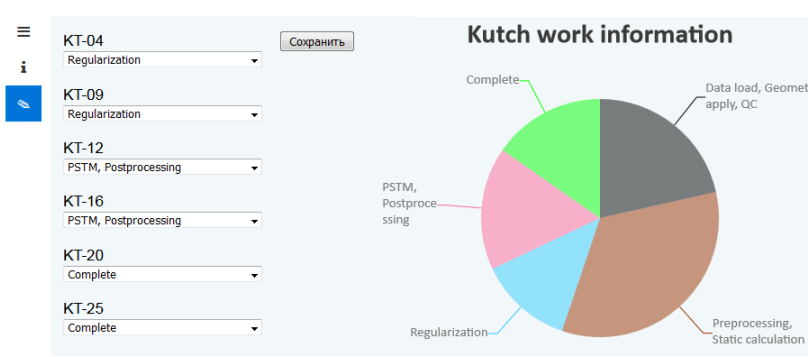

Figure 7. Dialog window of module to transfer data of $2 \mathrm{D}$ seismic lines processing status. (C) Loginov D.S., 2021

\subsection{Interactive map interface and its use}

The interactive map can be viewed on any web browser, as the map code is written in PHP. This code is used to connect to the database. The data visualisation is made with the help of the modern open source Leaflet library. The formation of the convention signs is provided by basic parameters of the display on the electronic map. The design of similar objects in the GIS project was used as a basis.

The interactive map series is aimed at performing both regional and prospecting and appraisal stages of geological exploration work. The resulting interactive maps allow real-time monitoring of field crews work, cameral data processing and interpretation, and other tasks.

The map is available in both desktop and mobile versions. This facilitates instant administrative and management decisions aimed at improving the efficiency of exploration planning and execution using base maps, which also include electronic topographic maps.

Target Horizon Inc. specialists use this principle to provide cartographic support for the comprehensive interpretation of geological and geophysical data in the license areas in Western and Eastern Siberia, Volga-Ural and Timano-Pechora provinces, in the Far East of Russian Federation, India, Colombia, Cuba and other regions of the world.

Web technologies has proved especially effective in organising the daily monitoring of the progress of field geodetic and seismic surveys. The dynamic form of content change involves the constant updating of the data table of geophysical observation points. As information becomes available, new objects are added to the interactive map, statistics and other background information are updated, allowing for promt changes in the planning of further work taking into account various factors (weather conditions, technical condition of equipment, readiness of field workers, etc.).

The interface of above mentioned interactive maps is shown in Figures 9 and 10.

\section{Conclusions}

Web maps represent a new means of communication between cross-industry specialists within a company, a means of optimising data access and a means of coordinating collaborative work to achieve different levels of tasks.
In general, there has been a positive experience with the use of web-based technologies in the geological exploration. Interactive cartographic geoportals and maps are widely used in geological mapping for analysis of the territory exploration geophysics methods, assessment of the geological situation of the study area, etc.

A high potential of interactive maps usage is observed for designing the network of survey profiles and for comparing diverse information about the geological structure, topographic features of the territory.

Application of open source software allows to form geoportals for special purposes, including mapping of surveyed areas and on-line monitoring of field work progress.

Further development of using web technologies for geological and geophysical works is focused on the improvement of current JavaScript libraries of data visualization, thematic focus of developed resources and providing data storage in archival systems. Active implementation of cloud technologies will enable not only to store archived data, but also to the remote interactive access to speed up the process of obtaining information and its use in research.

\section{Acknowledgements}

Author would like to thank the staff of the Department of Digital Cartography and GIS for help, support and improvement of the creation and preparation of interactive maps.

\section{References}

Bhukosh Geoportal. Geological Society of India. https://bhukosh.gsi.gov.in/Bhukosh/Public (accessed on 09 June 2019)

Leaflet. http://leafletjs.com (accessed on 11 June 2019)

Loginov D. S. (2019) Cartographic support of geophysical research: current state and prospects. Geodesy and Cartography, 80, 8, pp. 32-44 (In Russian) DOI: 10.22389/0016-7126-2019-950-8-32-44

Loginov, D.S. Specific features of using GIS atlases in the geophysical mapping // Proceedings of the 6th International Conference on Cartography and GIS, 1317 June 2016, Albena, Bulgaria. Eds: Bandrova T., Konecny M. - Pp. 615-623.

MBFSZ map server. https://map.mbfsz.gov.hu/ (accessed on 09 June 2019)

OneGeology.

http://portal.onegeology.org/OnegeologyGlobal/ (accessed on 09 June 2019)

Portale del Servizio Geologico d'Italia. http://sgi2.isprambiente.it/mapviewer/ (accessed on 11 June 2019)

PostgreSQL. https://www.postgresql.org/ (accessed on 11 June 2019) 


\section{Appendix}

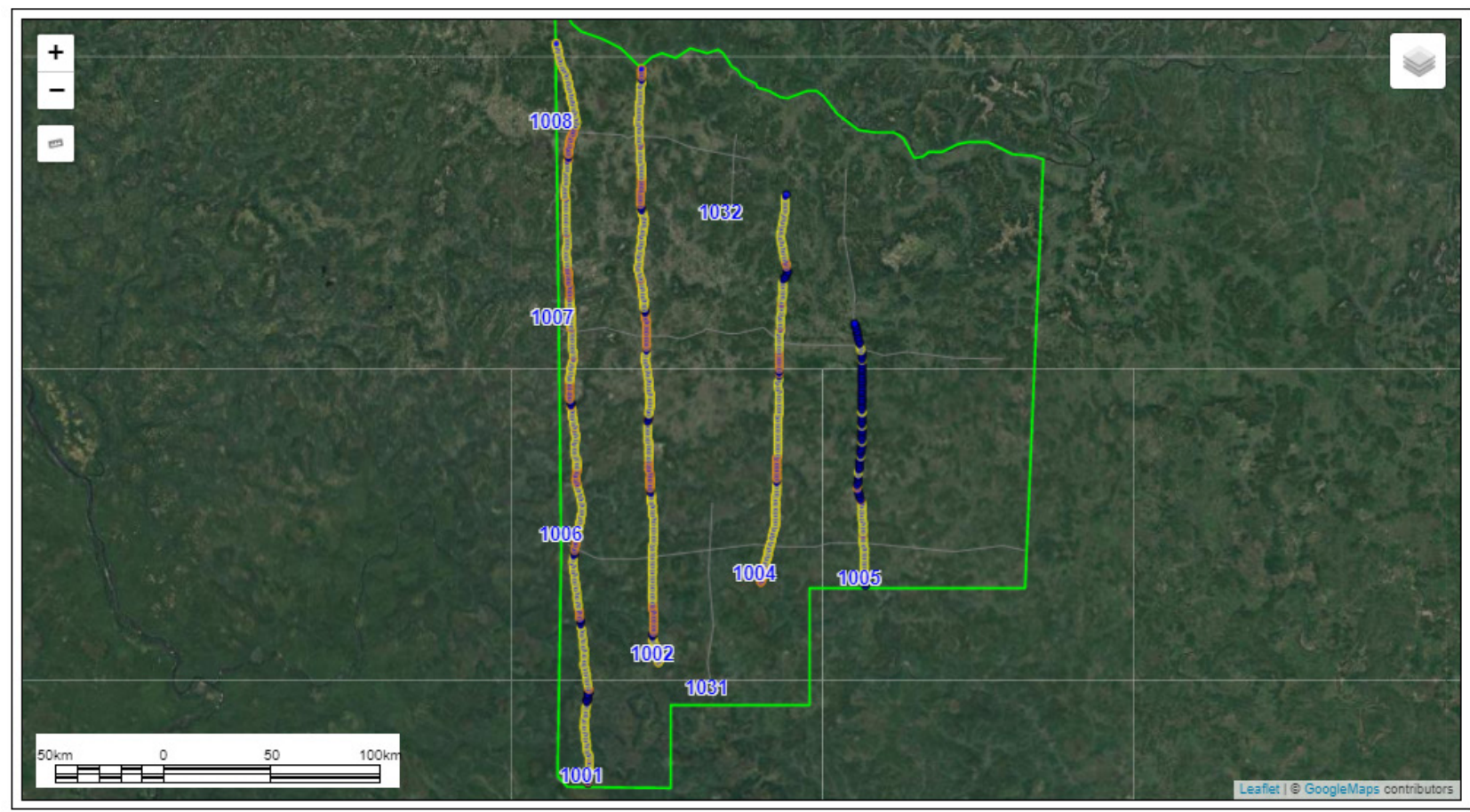

Figure 9. The interactive map for daily monitoring of field seismic acquisition $@$ Loginov D.S., 2021

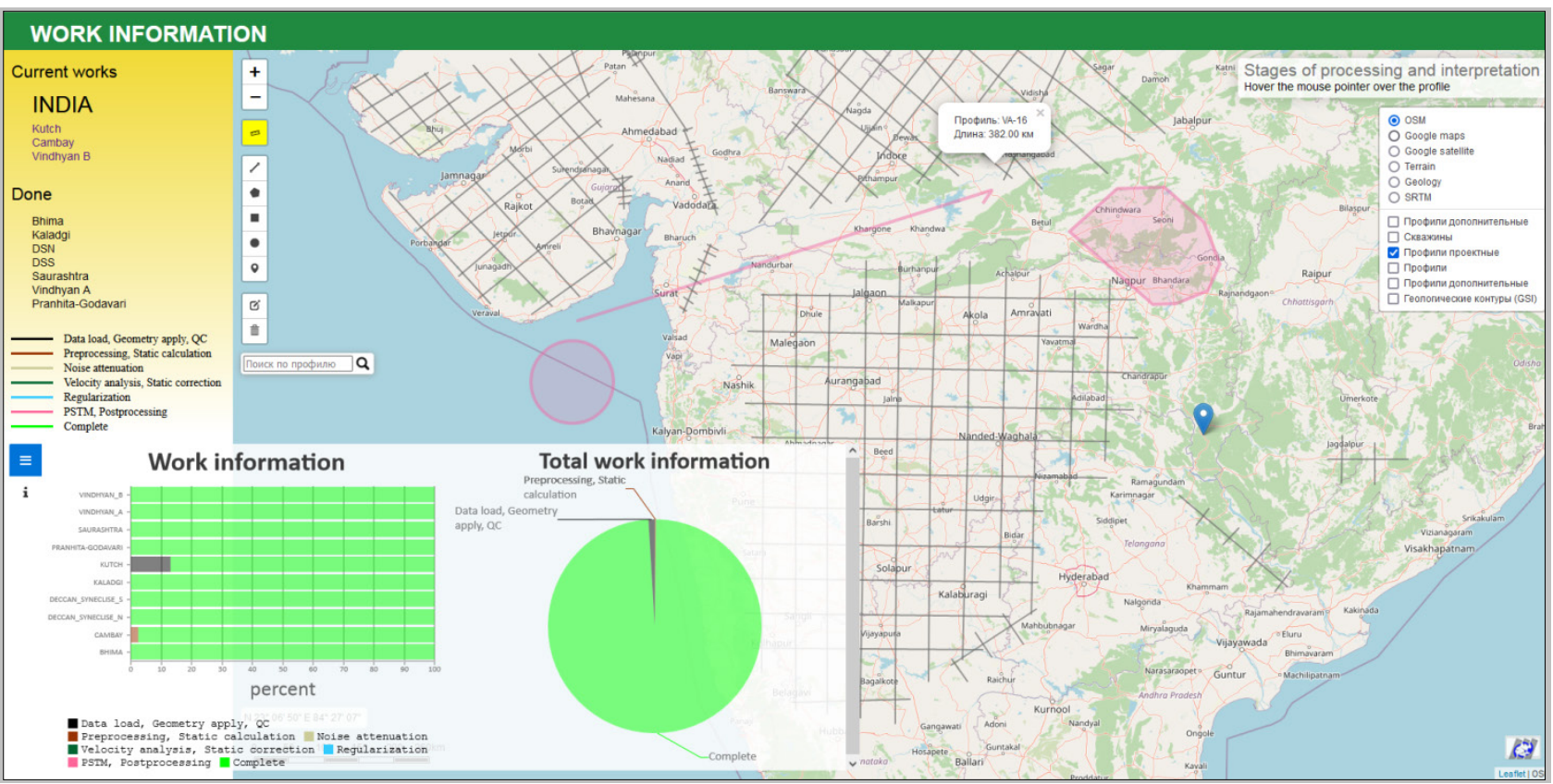

Figure 10. Work window of interactive map for monitoring of geological and geophysical data processing and interpretation (C) Loginov D.S., 2021 